\title{
A variable metric extension of the forward-backward-forward algorithm for monotone operators *
}

\author{
Bằng Công Vũ \\ UPMC Université Paris 06 \\ Laboratoire Jacques-Louis Lions - UMR CNRS 7598 \\ 75005 Paris, France \\ vu@ljll.math.upmc.fr
}

\begin{abstract}
We propose a variable metric extension of the forward-backward-forward algorithm for finding a zero of the sum of a maximally monotone operator and a monotone Lipschitzian operator in Hilbert spaces. In turn, this framework provides a variable metric splitting algorithm for solving monotone inclusions involving sums of composite operators. Monotone operator splitting methods recently proposed in the literature are recovered as special cases.
\end{abstract}

Keywords: variable metric, composite operator, duality, monotone inclusion, monotone operator, operator splitting, primal-dual algorithm

Mathematics Subject Classifications (2010) 47H05, 49M29, 49M27, 90C25

\section{Introduction}

A basic problem in applied monotone operator theory is to find a zero of a maximally monotone operator $A$ on a real Hilbert space $\mathcal{H}$. This problem can be solved by the proximal point algorithm proposed in [17] which requires only the resolvent of $A$, provided it is easy to implement numerically. In order to get more efficient proximal algorithms, some authors have proposed the use of variable metric or preconditioning in such algorithms [3, 5, 6, 10, 13, 15, 16].

This problem was then extended to the problem of finding a zero of the sum of a maximally monotone operator $A$ and a cocoercive operator $B$ (i.e., $B^{-1}$ is strongly monotone). In such

${ }^{*}$ This work was partially supported by Grant 102.01-2012.15 of the Vietnam National Foundation for Science and Technology Development (NAFOSTED). 
instances, the forward-backward splitting algorithm [1, 8, 12, 18] can be used. Recently, this algorithm has been investigated in the context of variable metric [11. In the case when $B$ is only Lipschitzian and not cocoercive, the problem can be solved by the forward-backward-forward splitting algorithm [4, 19. New applications of this basic algorithm to more complex monotone inclusions are presented in [4, 9].

In the present paper, we propose a variable metric version of the forward-backward-forward splitting algorithm. In Section 2, we recall notation and background on convex analysis and monotone operator theory. In Section 3, we present our variable metric forward-backward-forward splitting algorithm. In Section 4 , the results of Section 3 are used to develop a variable metric primal-dual algorithm for solving the type of composite inclusions considered in [9].

\section{Notation and background}

Throughout, $\mathcal{H}, \mathcal{G}$, and $\left(\mathcal{G}_{i}\right)_{1 \leq i \leq m}$ are real Hilbert spaces. Their scalar products and associated norms are respectively denoted by $\langle\cdot \mid \cdot\rangle$ and $\|\cdot\|$. We denote by $\mathcal{B}(\mathcal{H}, \mathcal{G})$ the space of bounded linear operators from $\mathcal{H}$ to $\mathcal{G}$. The adjoint of $L \in \mathcal{B}(\mathcal{H}, \mathcal{G})$ is denoted by $L^{*}$. We set $\mathcal{B}(\mathcal{H})=$ $\mathcal{B}(\mathcal{H}, \mathcal{H})$. The symbols $\rightarrow$ and $\rightarrow$ denote respectively weak and strong convergence, and Id denotes the identity operator, and $B(x ; \rho)$ denotes the closed ball of center $x \in \mathcal{H}$ and radius $\rho \in] 0,+\infty[$. The interior of $C \subset \mathcal{H}$ is denoted by $\operatorname{int} C$. We denote by $\ell_{+}^{1}(\mathbb{N})$ the set of summable sequences in $[0,+\infty[$.

Let $M_{1}$ and $M_{2}$ be self-adjoint operators in $\mathcal{B}(\mathcal{H})$, we write $M_{1} \succcurlyeq M_{2}$ if and only if $(\forall x \in$ $\mathcal{H})\left\langle M_{1} x \mid x\right\rangle \geq\left\langle M_{2} x \mid x\right\rangle$. Let $\left.\alpha \in\right] 0,+\infty[$. We set

$$
\mathcal{P}_{\alpha}(\mathcal{H})=\left\{M \in \mathcal{B}(\mathcal{H}) \mid M^{*}=M \quad \text { and } \quad M \succcurlyeq \alpha \operatorname{Id}\right\} .
$$

Moreover, for every $M \in \mathcal{P}_{\alpha}(\mathcal{H})$, we define respectively a scalar product and a norm by

$$
(\forall x \in \mathcal{H})(\forall y \in \mathcal{H}) \quad\langle x \mid y\rangle_{M}=\langle M x \mid y\rangle \quad \text { and } \quad\|x\|_{M}=\sqrt{\langle M x \mid x\rangle} .
$$

Let $A: \mathcal{H} \rightarrow 2^{\mathcal{H}}$ be a set-valued operator. The domain is $\operatorname{dom} A=\{x \in \mathcal{H} \mid A x \neq \varnothing\}$, and the graph of $A$ is gra $A=\{(x, u) \in \mathcal{H} \times \mathcal{H} \mid u \in A x\}$. The set of zeros of $A$ is zer $A=\{x \in \mathcal{H} \mid$ $0 \in A x\}$, and the range of $A$ is $\operatorname{ran} A=\{u \in \mathcal{H} \mid(\exists x \in \mathcal{H}) u \in A x\}$. The inverse of $A$ is $A^{-1}: \mathcal{H} \mapsto 2^{\mathcal{H}}: u \mapsto\{x \in \mathcal{H} \mid u \in A x\}$, and the resolvent of $A$ is

$$
J_{A}=(\mathrm{Id}+A)^{-1} \text {. }
$$

Moreover, $A$ is monotone if

$$
(\forall(x, y) \in \mathcal{H} \times \mathcal{H})(\forall(u, v) \in A x \times A y) \quad\langle x-y \mid u-v\rangle \geq 0,
$$

and maximally monotone if it is monotone and there exists no monotone operator $B: \mathcal{H} \rightarrow 2^{\mathcal{H}}$ such that gra $A \subset$ gra $B$ and $A \neq B$. We say that $A$ is uniformly monotone at $x \in \operatorname{dom} A$ if there exists an increasing function $\phi_{A}:[0,+\infty[\rightarrow[0,+\infty]$ vanishing only at 0 such that

$$
(\forall u \in A x)(\forall(y, v) \in \operatorname{gra} A) \quad\langle x-y \mid u-v\rangle \geq \phi_{A}(\|x-y\|) .
$$




\section{Variable metric forward-backward-forward splitting algorithm}

The forward-backward-forward splitting algorithm was first proposed in [19] to solve inclusion involving the sum of a maximally monotone operator and a monotone Lipschitzian operator. In [4], it was revisited to include computational errors. Below, we extend it to a variable metric setting.

Theorem 3.1 Let $\mathcal{K}$ be a real Hilbert space with the scalar product $\langle\langle\langle\cdot \mid \cdot\rangle\rangle\rangle$ and the associated norm \|\|$\cdot|\|| \mid$. Let $\alpha$ and $\beta$ be in $] 0,+\infty\left[\right.$, let $\left(\eta_{n}\right)_{n \in \mathbb{N}}$ be a sequence in $\ell_{+}^{1}(\mathbb{N})$, and let $\left(\boldsymbol{U}_{n}\right)_{n \in \mathbb{N}}$ be a sequence in $\mathcal{B}(\mathcal{K})$ such that

$$
\mu=\sup _{n \in \mathbb{N}}\left\|\boldsymbol{U}_{n}\right\|<+\infty \quad \text { and } \quad\left(1+\eta_{n}\right) \boldsymbol{U}_{n+1} \succcurlyeq \boldsymbol{U}_{n} \in \mathcal{P}_{\alpha}(\mathcal{K}) .
$$

Let $\boldsymbol{A}: \mathcal{K} \rightarrow 2^{\mathcal{K}}$ be maximally monotone, let $\boldsymbol{B}: \mathcal{K} \rightarrow \mathcal{K}$ be a monotone and $\beta$-Lipschitzian operator on $\mathcal{K}$ such that $\operatorname{zer}(\boldsymbol{A}+\boldsymbol{B}) \neq \varnothing$. Let $\left(\boldsymbol{a}_{n}\right)_{n \in \mathbb{N}},\left(\boldsymbol{b}_{n}\right)_{n \in \mathbb{N}}$, and $\left(\boldsymbol{c}_{n}\right)_{n \in \mathbb{N}}$ be absolutely summable sequences in $\mathcal{K}$. Let $\boldsymbol{x}_{0} \in \mathcal{K}$, let $\left.\varepsilon \in\right] 0,1 /(\beta \mu+1)\left[\right.$, let $\left(\gamma_{n}\right)_{n \in \mathbb{N}}$ be a sequence in $[\varepsilon,(1-\varepsilon) /(\beta \mu)]$, and set

$$
(\forall n \in \mathbb{N}) \quad\left[\begin{array}{l}
\boldsymbol{y}_{n}=\boldsymbol{x}_{n}-\gamma_{n} \boldsymbol{U}_{n}\left(\boldsymbol{B} \boldsymbol{x}_{n}+\boldsymbol{a}_{n}\right) \\
\boldsymbol{p}_{n}=J_{\gamma_{n} \boldsymbol{U}_{n} \boldsymbol{A}} \boldsymbol{y}_{n}+\boldsymbol{b}_{n} \\
\boldsymbol{q}_{n}=\boldsymbol{p}_{n}-\gamma_{n} \boldsymbol{U}_{n}\left(\boldsymbol{B} \boldsymbol{p}_{n}+\boldsymbol{c}_{n}\right) \\
\boldsymbol{x}_{n+1}=\boldsymbol{x}_{n}-\boldsymbol{y}_{n}+\boldsymbol{q}_{n} .
\end{array}\right.
$$

Then the following hold for some $\overline{\boldsymbol{x}} \in \operatorname{zer}(\boldsymbol{A}+\boldsymbol{B})$.

(i) $\left.\sum_{n \in \mathbb{N}}\left|\left\||| \boldsymbol{x}_{n}-\boldsymbol{p}_{n}||\right\|^{2}<+\infty\right.$ and $\left.\sum_{n \in \mathbb{N}}\right|\left\|\left|\boldsymbol{y}_{n}-\boldsymbol{q}_{n}\right|\right\|\right|^{2}<+\infty$.

(ii) $\boldsymbol{x}_{n} \rightarrow \overline{\boldsymbol{x}}$ and $\boldsymbol{p}_{n} \rightarrow \overline{\boldsymbol{x}}$.

(iii) Suppose that one of the following is satisfied:

(a) $\underline{\lim } d_{\operatorname{zer}(\boldsymbol{A}+\boldsymbol{B})}\left(\boldsymbol{x}_{n}\right)=0$.

(b) $\boldsymbol{A}+\boldsymbol{B}$ is demiregular (see [1, Definition 2.3]) at $\overline{\boldsymbol{x}}$.

(c) $\boldsymbol{A}$ or $\boldsymbol{B}$ is uniformly monotone at $\overline{\boldsymbol{x}}$.

(d) $\operatorname{int} \operatorname{zer}(\boldsymbol{A}+\boldsymbol{B}) \neq \varnothing$ and there exists $\left(\nu_{n}\right)_{n \in \mathbb{N}} \in \ell_{+}^{1}(\mathbb{N})$ such that $(\forall n \in \mathbb{N})\left(1+\nu_{n}\right) \boldsymbol{U}_{n} \succeq$ $\boldsymbol{U}_{n+1}$.

Then $\boldsymbol{x}_{n} \rightarrow \overline{\boldsymbol{x}}$ and $\boldsymbol{p}_{n} \rightarrow \overline{\boldsymbol{x}}$

Proof. It follows from [11, Lemma 3.7] that the sequences $\left(\boldsymbol{x}_{n}\right)_{n \in \mathbb{N}},\left(\boldsymbol{y}_{n}\right)_{n \in \mathbb{N}},\left(\boldsymbol{p}_{n}\right)_{n \in \mathbb{N}}$ and $\left(\boldsymbol{q}_{n}\right)_{n \in \mathbb{N}}$ are well defined. Moreover, using [10, Lemma 2.1(i)(ii)] and (3.1), we obtain

$$
\left(\forall\left(\boldsymbol{z}_{n}\right)_{n \in \mathbb{N}} \in \mathcal{K}^{\mathbb{N}}\right) \quad \sum_{n \in \mathbb{N}}\|\| \boldsymbol{z}_{n}||\left\|<+\infty \Leftrightarrow \sum_{n \in \mathbb{N}}\left|\left\|\mid \boldsymbol{z}_{n}\right\| \|_{\boldsymbol{U}_{n}^{-1}}<+\infty\right.\right.
$$

and

$$
\left(\forall\left(\boldsymbol{z}_{n}\right)_{n \in \mathbb{N}} \in \mathcal{K}^{\mathbb{N}}\right) \quad \sum_{n \in \mathbb{N}}\|\| \boldsymbol{z}_{n}\|\|\left|<+\infty \Leftrightarrow \sum_{n \in \mathbb{N}}\right|\left\|\mid \boldsymbol{z}_{n}\right\|\|\|_{\boldsymbol{U}_{n}}<+\infty .
$$


Let us set

$$
(\forall n \in \mathbb{N}) \quad\left\{\begin{array} { l } 
{ \widetilde { \boldsymbol { y } } _ { n } = \boldsymbol { x } _ { n } - \gamma _ { n } \boldsymbol { U } _ { n } \boldsymbol { B } \boldsymbol { x } _ { n } } \\
{ \widetilde { \boldsymbol { p } } _ { n } = J _ { \gamma _ { n } \boldsymbol { U } _ { n } \boldsymbol { A } } \widetilde { \boldsymbol { y } } _ { n } } \\
{ \widetilde { \boldsymbol { q } } _ { n } = \widetilde { \boldsymbol { p } } _ { n } - \gamma _ { n } \boldsymbol { U } _ { n } \boldsymbol { B } \widetilde { \boldsymbol { p } } _ { n } } \\
{ \widetilde { \boldsymbol { x } } _ { n + 1 } = \boldsymbol { x } _ { n } - \widetilde { \boldsymbol { y } } _ { n } + \widetilde { \boldsymbol { q } } _ { n } , }
\end{array} \quad \text { and } \quad \left\{\begin{array}{l}
\boldsymbol{u}_{n}=\gamma_{n}^{-1} \boldsymbol{U}_{n}^{-1}\left(\boldsymbol{x}_{n}-\widetilde{\boldsymbol{p}}_{n}\right)+\boldsymbol{B} \widetilde{\boldsymbol{p}}_{n}-\boldsymbol{B} \boldsymbol{x}_{n} \\
\boldsymbol{e}_{n}=\widetilde{\boldsymbol{x}}_{n+1}-\boldsymbol{x}_{n+1} \\
\boldsymbol{d}_{n}=\boldsymbol{q}_{n}-\widetilde{\boldsymbol{q}}_{n}+\widetilde{\boldsymbol{y}}_{n}-\boldsymbol{y}_{n} .
\end{array}\right.\right.
$$

Then (3.5) yields

$$
(\forall n \in \mathbb{N}) \quad \boldsymbol{u}_{n}=\gamma_{n}^{-1} \boldsymbol{U}_{n}^{-1}\left(\widetilde{\boldsymbol{y}}_{n}-\widetilde{\boldsymbol{p}}_{n}\right)+\boldsymbol{B} \widetilde{\boldsymbol{p}}_{n} \in \boldsymbol{A} \widetilde{\boldsymbol{p}}_{n}+\boldsymbol{B} \widetilde{\boldsymbol{p}}_{n},
$$

and (3.5), (3.2), Lemma [11, Lemma 3.7(ii)], and the Lipschitzianity of $\boldsymbol{B}$ on $\mathcal{K}$ yield

$$
(\forall n \in \mathbb{N})\left\{\begin{array}{l}
|||| \boldsymbol{y}_{n}-\widetilde{\boldsymbol{y}}_{n}||||_{\boldsymbol{U}_{n}^{-1}} \leq(\beta \mu)^{-1}|||| \boldsymbol{a}_{n}||||_{\boldsymbol{U}_{n}} \\
|||| \boldsymbol{p}_{n}-\widetilde{\boldsymbol{p}}_{n}||||_{\boldsymbol{U}_{n}^{-1}} \leq|||| \boldsymbol{b}_{n}||||_{\boldsymbol{U}_{n}^{-1}}+(\beta \mu)^{-1}|||| \boldsymbol{a}_{n}||||_{\boldsymbol{U}_{n}} \\
|||| \boldsymbol{q}_{n}-\left.\widetilde{\boldsymbol{q}}_{n}||\right|_{\boldsymbol{U}_{n}^{-1}} \leq 2\left(\left.|||| \boldsymbol{b}_{n}||\right|_{\boldsymbol{U}_{n}^{-1}}+(\beta \mu)^{-1}|||| \boldsymbol{a}_{n}||||_{\boldsymbol{U}_{n}}\right)+(\beta \mu)^{-1}|||| \boldsymbol{c}_{n}||||_{\boldsymbol{U}_{n}} .
\end{array}\right.
$$

Since $\left(\boldsymbol{a}_{n}\right)_{n \in \mathbb{N}},\left(\boldsymbol{b}_{n}\right)_{n \in \mathbb{N}}$, and $\left(\boldsymbol{c}_{n}\right)_{n \in \mathbb{N}}$ are absolutely summable sequences in $\mathcal{K}$, we derive from (3.3), (3.4), (3.5), and (3.7) that

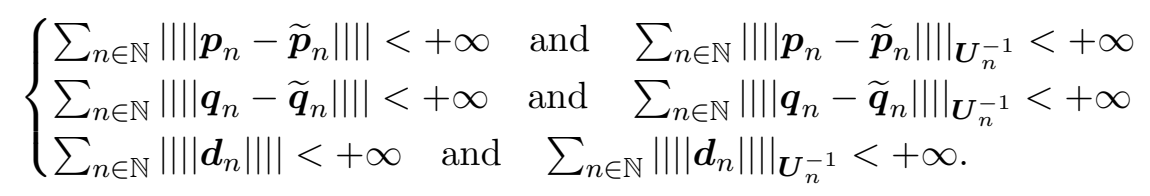

Now, let $\boldsymbol{x} \in \operatorname{zer}(\boldsymbol{A}+\boldsymbol{B})$. Then, for every $n \in \mathbb{N},\left(\boldsymbol{x},-\gamma_{n} \boldsymbol{U}_{n} \boldsymbol{B} \boldsymbol{x}\right) \in \operatorname{gra}\left(\gamma_{n} \boldsymbol{U}_{n} \boldsymbol{A}\right)$ and (3.5) yields $\left(\widetilde{\boldsymbol{p}}_{n}, \widetilde{\boldsymbol{y}}_{n}-\widetilde{\boldsymbol{p}}_{n}\right) \in \operatorname{gra}\left(\gamma_{n} \boldsymbol{U}_{n} \boldsymbol{A}\right)$. Hence, by monotonicity of $\boldsymbol{U}_{n} \boldsymbol{A}$ with respect to the scalar product $\langle\langle\langle\cdot|\cdot|\rangle\rangle\rangle_{\boldsymbol{U}_{n}^{-1}}$, we have $\left\langle\left\langle\left\langle\widetilde{\boldsymbol{p}}_{n}-\boldsymbol{x} \mid \widetilde{\boldsymbol{p}}_{n}-\widetilde{\boldsymbol{y}}_{n}-\gamma_{n} \boldsymbol{U}_{n} \boldsymbol{B} \boldsymbol{x}\right\rangle\right\rangle\right\rangle_{\boldsymbol{U}_{n}^{-1}} \leq 0$. Moreover, by monotonicity of $\boldsymbol{U}_{n} \boldsymbol{B}^{n}$ with respect to the scalar product $\left\langle\langle\langle\cdot \mid \cdot \cdot\rangle\rangle_{\boldsymbol{U}_{n}^{-1}}\right.$, we also have $\left\langle\left\langle\left\langle\widetilde{\boldsymbol{p}}_{n}-\boldsymbol{x} \mid \gamma_{n} \boldsymbol{U}_{n} \boldsymbol{B} \boldsymbol{x}-\gamma_{n} \boldsymbol{U}_{n} \boldsymbol{B} \widetilde{\boldsymbol{p}}_{n}\right\rangle\right\rangle\right\rangle_{\boldsymbol{U}_{n}^{-1}} \leq 0$. By adding the last two inequalities, we obtain

$$
(\forall n \in \mathbb{N}) \quad\left\langle\left\langle\left\langle\widetilde{\boldsymbol{p}}_{n}-\boldsymbol{x} \mid \widetilde{\boldsymbol{p}}_{n}-\widetilde{\boldsymbol{y}}_{n}-\gamma_{n} \boldsymbol{U}_{n} \boldsymbol{B} \widetilde{\boldsymbol{p}}_{n}\right\rangle\right\rangle\right\rangle_{\boldsymbol{U}_{n}^{-1}} \leq 0 .
$$

In turn, we derive from (3.5) that

$$
\begin{aligned}
&(\forall n \in \mathbb{N}) \quad 2 \gamma_{n}\left\langle\left\langle\left\langle\widetilde{\boldsymbol{p}}_{n}-\boldsymbol{x} \mid \boldsymbol{U}_{n} \boldsymbol{B} \boldsymbol{x}_{n}-\boldsymbol{U}_{n} \boldsymbol{B} \widetilde{\boldsymbol{p}}_{n}\right\rangle\right\rangle\right\rangle_{\boldsymbol{U}_{n}^{-1}} \\
&=2\left\langle\left\langle\left\langle\widetilde{\boldsymbol{p}}_{n}-\boldsymbol{x} \mid \widetilde{\boldsymbol{p}}_{n}-\widetilde{\boldsymbol{y}}_{n}-\gamma_{n} \boldsymbol{U}_{n} \boldsymbol{B} \widetilde{\boldsymbol{p}}_{n}\right\rangle\right\rangle\right\rangle_{\boldsymbol{U}_{n}^{-1}} \\
&+2\left\langle\left\langle\left\langle\widetilde{\boldsymbol{p}}_{n}-\boldsymbol{x} \mid \gamma_{n} \boldsymbol{U}_{n} \boldsymbol{B} \boldsymbol{x}_{n}+\widetilde{\boldsymbol{y}}_{n}-\widetilde{\boldsymbol{p}}_{n}\right\rangle\right\rangle\right\rangle_{\boldsymbol{U}_{n}^{-1}} \\
& \leq 2\left\langle\left\langle\left\langle\widetilde{\boldsymbol{p}}_{n}-\boldsymbol{x} \mid \gamma_{n} \boldsymbol{U}_{n} \boldsymbol{B} \boldsymbol{x}_{n}+\widetilde{\boldsymbol{y}}_{n}-\widetilde{\boldsymbol{p}}_{n}\right\rangle\right\rangle\right\rangle_{\boldsymbol{U}_{n}^{-1}} \\
&= 2\left\langle\left\langle\left\langle\widetilde{\boldsymbol{p}}_{n}-\boldsymbol{x} \mid \boldsymbol{x}_{n}-\widetilde{\boldsymbol{p}}_{n}\right\rangle\right\rangle\right\rangle_{\boldsymbol{U}_{n}^{-1}} \\
&=|||| \boldsymbol{x}_{n}-\boldsymbol{x}\left|\left\|\left.\right|_{\boldsymbol{U}_{n}^{-1}} ^{2}-|||| \widetilde{\boldsymbol{p}}_{n}-\boldsymbol{x}||\right\|_{\boldsymbol{U}_{n}^{-1}}^{2}-\right|||\left|\boldsymbol{x}_{n}-\widetilde{\boldsymbol{p}}_{n}\right| \|_{\boldsymbol{U}_{n}^{-1}}^{2} .
\end{aligned}
$$


Hence, using (3.5), (3.10), the $\beta$-Lipschitz continuity of $\boldsymbol{B}$, (3.1), and [10, Lemma 2.1(ii)], for every $n \in \mathbb{N}$, we obtain

$$
\begin{aligned}
& \left.\left\||| \widetilde{\boldsymbol{x}}_{n+1}-\boldsymbol{x}\right\|\left\|_{\boldsymbol{U}_{n}^{-1}}^{2}=\right\|\left\|\left|\widetilde{\boldsymbol{q}}_{n}+\boldsymbol{x}_{n}-\widetilde{\boldsymbol{y}}_{n}-\boldsymbol{x}\right|\right\|\right|_{\boldsymbol{U}_{n}^{-1}} ^{2} \\
& =\left\|||\left(\widetilde{\boldsymbol{p}}_{n}-\boldsymbol{x}\right)+\gamma_{n} \boldsymbol{U}_{n}\left(\boldsymbol{B} \boldsymbol{x}_{n}-\boldsymbol{B} \widetilde{\boldsymbol{p}}_{n}\right) \mid\right\| \|_{\boldsymbol{U}_{n}^{-1}}^{2} \\
& =|||| \widetilde{\boldsymbol{p}}_{n}-\boldsymbol{x}|| \|_{\boldsymbol{U}_{n}^{-1}}^{2}+2 \gamma_{n}\left\langle\left\langle\left\langle\widetilde{\boldsymbol{p}}_{n}-\boldsymbol{x} \mid \boldsymbol{B} \boldsymbol{x}_{n}-\boldsymbol{B} \widetilde{\boldsymbol{p}}_{n}\right\rangle\right\rangle\right\rangle \\
& +\gamma_{n}^{2}|||| \boldsymbol{U}_{n}\left(\boldsymbol{B} \boldsymbol{x}_{n}-\boldsymbol{B} \widetilde{\boldsymbol{p}}_{n}\right) \mid\|\|_{\boldsymbol{U}_{n}^{-1}}^{2} \\
& \leq|||| \boldsymbol{x}_{n}-\boldsymbol{x}||\left\|_{\boldsymbol{U}_{n}^{-1}}^{2}-|||| \boldsymbol{x}_{n}-\left.\widetilde{\boldsymbol{p}}_{n}||\right|_{\boldsymbol{U}_{n}^{-1}} ^{2}+\gamma_{n}^{2} \mu \beta^{2}|||| \boldsymbol{x}_{n}-\widetilde{\boldsymbol{p}}_{n}||\right\|^{2} \\
& \leq|||| \boldsymbol{x}_{n}-\left.\boldsymbol{x}||\right|_{\boldsymbol{U}_{n}^{-1}} ^{2}-\mu^{-1}|||| \boldsymbol{x}_{n}-\left.\widetilde{\boldsymbol{p}}_{n}||\right|^{2}+\gamma_{n}^{2} \mu \beta^{2}|||| \boldsymbol{x}_{n}-\widetilde{\boldsymbol{p}}_{n}||||^{2} .
\end{aligned}
$$

Hence, it follows from (3.1) and [10, Lemma 2.1(i)] that

$$
(\forall n \in \mathbb{N}) \quad\left\||| \widetilde{\boldsymbol{x}}_{n+1}-\boldsymbol{x}\left|\left\|\left.\right|_{\boldsymbol{U}_{n+1}^{-1}} ^{2} \leq\left(1+\eta_{n}\right)|||| \boldsymbol{x}_{n}-\boldsymbol{x}||\right\|_{\boldsymbol{U}_{n}^{-1}}^{2}-\mu^{-1}\left(1-\gamma_{n}^{2} \beta^{2} \mu^{2}\right)\right|||\left|\boldsymbol{x}_{n}-\widetilde{\boldsymbol{p}}_{n}\right| \mid\right\|^{2} .
$$

Consequently,

$$
(\forall n \in \mathbb{N}) \quad \||| \widetilde{\boldsymbol{x}}_{n+1}-\boldsymbol{x}||||_{\boldsymbol{U}_{n+1}^{-1}} \leq\left(1+\eta_{n}\right)|||| \boldsymbol{x}_{n}-\left.\boldsymbol{x}||\right|_{\boldsymbol{U}_{n}^{-1}}
$$

For every $n \in \mathbb{N}$, set

$$
\varepsilon_{n}=\sqrt{\mu \alpha^{-1}}\left(2\left(\left.|||| \boldsymbol{b}_{n}||\right|_{\boldsymbol{U}_{n}^{-1}}+\left.(\beta \mu)^{-1}|||| \boldsymbol{a}_{n}||\right|_{\boldsymbol{U}_{n}}\right)+(\beta \mu)^{-1}|||| \boldsymbol{c}_{n}||||_{\boldsymbol{U}_{n}}+(\beta \mu)^{-1}|||| \boldsymbol{a}_{n}|| \|_{\boldsymbol{U}_{n}}\right) .
$$

Then $\left(\varepsilon_{n}\right)_{n \in \mathbb{N}}$ is summable by (3.3) and (3.4). We derive from [10, Lemma 2.1(ii)(iii)], and (3.8) that

$$
\begin{aligned}
(\forall n \in \mathbb{N}) \quad \||| \boldsymbol{e}_{n}||||_{\boldsymbol{U}_{n+1}^{-1}} & =|||| \widetilde{\boldsymbol{x}}_{n+1}-\boldsymbol{x}_{n+1}||||_{\boldsymbol{U}_{n+1}^{-1}} \\
& \leq \sqrt{\alpha^{-1}}|||| \widetilde{\boldsymbol{x}}_{n+1}-\boldsymbol{x}_{n+1}|||| \\
& \leq \sqrt{\mu \alpha^{-1}}|||| \widetilde{\boldsymbol{x}}_{n+1}-\left.\boldsymbol{x}_{n+1}||\right|_{\boldsymbol{U}_{n}^{-1}} \\
& \leq \sqrt{\mu \alpha^{-1}}\left(|||| \widetilde{\boldsymbol{y}}_{n}-\boldsymbol{y}_{n}||||_{\boldsymbol{U}_{n}^{-1}}+|||| \widetilde{\boldsymbol{q}}_{n}-\left.\boldsymbol{q}_{n}||\right|_{\boldsymbol{U}_{n}^{-1}}\right) \\
& \leq \varepsilon_{n} .
\end{aligned}
$$

In turn, we derive from (3.13) that

$$
\begin{aligned}
& (\forall n \in \mathbb{N}) \quad\left\||| \boldsymbol{x}_{n+1}-\left.\boldsymbol{x}||\right|_{\boldsymbol{U}_{n+1}^{-1}} \leq|||| \widetilde{\boldsymbol{x}}_{n+1}-\boldsymbol{x}||\right\|_{\boldsymbol{U}_{n+1}^{-1}}+|||| \widetilde{\boldsymbol{x}}_{n+1}-\boldsymbol{x}_{n+1}||||_{\boldsymbol{U}_{n+1}^{-1}} \\
& \leq|||| \widetilde{\boldsymbol{x}}_{n+1}-\boldsymbol{x}\|\|_{\boldsymbol{U}_{n+1}^{-1}}+\varepsilon_{n} \\
& \leq\left(1+\eta_{n}\right)|||| \boldsymbol{x}_{n}-\boldsymbol{x}|| \|_{\boldsymbol{U}_{n}^{-1}}+\varepsilon_{n} \text {. }
\end{aligned}
$$

This shows that $\left(\boldsymbol{x}_{n}\right)_{n \in \mathbb{N}}$ is $|\cdot|$-quasi-Fejér monotone with respect to the target set $\operatorname{zer}(\boldsymbol{A}+\boldsymbol{B})$ relative to $\left(\boldsymbol{U}_{n}^{-1}\right)_{n \in \mathbb{N}}$. Moreover, by [10, Proposition 3.2], $\left(\||| \boldsymbol{x}_{n}-\left.\boldsymbol{x}||\right|_{\boldsymbol{U}_{n}^{-1}}\right)_{n \in \mathbb{N}}$ is bounded. In turn, since $\boldsymbol{B}$ and $\left(J_{\gamma_{n} \boldsymbol{U}_{n} \boldsymbol{A}}\right)_{n \in \mathbb{N}}$ are Lipschitzian, and $(\forall n \in \mathbb{N}) \boldsymbol{x}=J_{\gamma_{n} \boldsymbol{U}_{n} \boldsymbol{A}}\left(\boldsymbol{x}-\gamma_{n} \boldsymbol{U}_{n} \boldsymbol{B} \boldsymbol{x}\right)$, we deduce from (3.5) that $\left(\widetilde{\boldsymbol{y}}_{n}\right)_{n \in \mathbb{N}},\left(\widetilde{\boldsymbol{p}}_{n}\right)_{n \in \mathbb{N}}$, and $\left(\widetilde{\boldsymbol{q}}_{n}\right)_{n \in \mathbb{N}}$ are bounded. Therefore,

$$
\tau=\sup _{n \in \mathbb{N}}\left\{|||| \boldsymbol{x}_{n}-\widetilde{\boldsymbol{y}}_{n}+\widetilde{\boldsymbol{q}}_{n}-\boldsymbol{x}||||_{\boldsymbol{U}_{n}^{-1}},|||| \boldsymbol{x}_{n}-\left.\boldsymbol{x}||\right|_{\boldsymbol{U}_{n}^{-1}}, 1+\eta_{n}\right\}<+\infty
$$


Hence, using (3.5), Cauchy-Schwarz for the norms $\left(|||| \cdot|| \|_{U_{n}^{-1}}\right)_{n \in \mathbb{N}}$, and (3.11), we get

$$
\begin{aligned}
& (\forall n \in \mathbb{N}) \quad\left\||| \boldsymbol{x}_{n+1}-\boldsymbol{x}\right\|\left\|_{\boldsymbol{U}_{n}^{-1}}^{2}=\right\|\left\|\boldsymbol{x}_{n}-\boldsymbol{y}_{n}+\boldsymbol{q}_{n}-\boldsymbol{x}|\||_{\boldsymbol{U}_{n}^{-1}}^{2}\right. \\
& =\|\| \widetilde{\boldsymbol{q}}_{n}+\boldsymbol{x}_{n}-\widetilde{\boldsymbol{y}}_{n}-\boldsymbol{x}+\boldsymbol{d}_{n}\|\|_{\boldsymbol{U}_{n}^{-1}}^{2} \\
& \leq|||| \widetilde{\boldsymbol{q}}_{n}+\boldsymbol{x}_{n}-\widetilde{\boldsymbol{y}}_{n}-\boldsymbol{x}||||_{\boldsymbol{U}_{n}^{-1}}^{2}+2 \tau|||| \boldsymbol{d}_{n}||||_{\boldsymbol{U}_{n}^{-1}}+|||| \boldsymbol{d}_{n}||||_{\boldsymbol{U}_{n}^{-1}}^{2} \\
& \leq|||| \boldsymbol{x}_{n}-\left.\boldsymbol{x}||\right|_{\boldsymbol{U}_{n}^{-1}} ^{2}-\mu^{-1}\left(1-\gamma_{n}^{2} \beta^{2} \mu^{2}\right)|||| \boldsymbol{x}_{n}-\widetilde{\boldsymbol{p}}_{n}\|\|^{2}+\varepsilon_{1, n} \text {, }
\end{aligned}
$$

where $(\forall n \in \mathbb{N}) \varepsilon_{1, n}=2 \tau|||| \boldsymbol{d}_{n}||\left\|_{\boldsymbol{U}_{n}^{-1}}+\right\||| \boldsymbol{d}_{n}|| \|_{\boldsymbol{U}_{n}^{-1}}^{2}$. In turn, for every $n \in \mathbb{N}$, by (3.1) and [10, Lemma 2.1(i)],

$$
\begin{aligned}
\|\| \boldsymbol{x}_{n+1}-\boldsymbol{x}\|\|_{\boldsymbol{U}_{n+1}^{-1}}^{2} & \leq\left(1+\eta_{n}\right) \mid\|\| \boldsymbol{x}_{n+1}-\boldsymbol{x}\|\|_{\boldsymbol{U}_{n}^{-1}}^{2} \\
& \leq\left|\left\||| \boldsymbol{x}_{n}-\boldsymbol{x}\left|\left\|\left.\right|_{\boldsymbol{U}_{n}^{-1}} ^{2}-\mu^{-1}\left(1-\gamma_{n}^{2} \beta^{2} \mu^{2}\right)\left|\left\||| \boldsymbol{x}_{n}-\widetilde{\boldsymbol{p}}_{n}|\||^{2}+\tau \varepsilon_{1, n}+\tau^{2} \eta_{n} .\right.\right.\right.\right.\right.\right.
\end{aligned}
$$

Since $\left(\tau \varepsilon_{1, n}+\tau^{2} \eta_{n}\right)_{n \in \mathbb{N}} \in \ell_{+}^{1}(\mathbb{N})$ by (3.8), it follows from [7, Lemma 3.1] that

$$
\sum_{n \in \mathbb{N}}|||| \boldsymbol{x}_{n}-\left.\widetilde{\boldsymbol{p}}_{n}||\right|^{2}<+\infty
$$

(i): It follows from (3.20) and (3.8) that

$$
\sum_{n \in \mathbb{N}}|||| \boldsymbol{x}_{n}-\left.\boldsymbol{p}_{n}||\right|^{2} \leq\left. 2 \sum_{n \in \mathbb{N}}\left|\|\| \boldsymbol{x}_{n}-\widetilde{\boldsymbol{p}}_{n}\right|||\right|^{2}+\left.2 \sum_{n \in \mathbb{N}}\left|\left\||| \boldsymbol{p}_{n}-\widetilde{\boldsymbol{p}}_{n}\right\|\right|\right|^{2}<+\infty .
$$

Furthermore, we derive from (3.8) and (3.5) that

$$
\begin{aligned}
\sum_{n \in \mathbb{N}}\|\| \boldsymbol{y}_{n}-\left.\boldsymbol{q}_{n}\|\|\right|^{2} & =\sum_{n \in \mathbb{N}}\|\| \widetilde{\boldsymbol{q}}_{n}-\widetilde{\boldsymbol{y}}_{n}+\boldsymbol{d}_{n}\|\| \|^{2} \\
& =\sum_{n \in \mathbb{N}}\|\| \widetilde{\boldsymbol{p}}_{n}-\boldsymbol{x}_{n}+\gamma_{n} \boldsymbol{U}_{n}\left(\boldsymbol{B} \boldsymbol{x}_{n}-\boldsymbol{B} \widetilde{\boldsymbol{p}}_{n}\right)+\boldsymbol{d}_{n}\|\|^{2} \\
& \leq 3\left(\sum_{n \in \mathbb{N}}\|\| \boldsymbol{x}_{n}-\left.\widetilde{\boldsymbol{p}}_{n}\|\|\right|^{2}+\|\| \gamma_{n} \boldsymbol{U}_{n}\left(\boldsymbol{B} \boldsymbol{x}_{n}-\boldsymbol{B} \widetilde{\boldsymbol{p}}_{n}\right)\|\|^{2}+\|\| \boldsymbol{d}_{n}\|\|^{2}\right) \\
& <+\infty .
\end{aligned}
$$

(ii) Let $\boldsymbol{x}$ be a weak cluster point of $\left(\boldsymbol{x}_{n}\right)_{n \in \mathbb{N}}$. Then there exists a subsequence $\left(\boldsymbol{x}_{k_{n}}\right)_{n \in \mathbb{N}}$ that converges weakly to $\boldsymbol{x}$. Therefore $\widetilde{\boldsymbol{p}}_{k_{n}} \rightarrow \boldsymbol{x}$ by (3.20). Furthermore, it follows from (3.5) that $\boldsymbol{u}_{k_{n}} \rightarrow 0$. Hence, since $(\forall n \in \mathbb{N})\left(\widetilde{\boldsymbol{p}}_{k_{n}}, \boldsymbol{u}_{k_{n}}\right) \in \operatorname{gra}(\boldsymbol{A}+\boldsymbol{B})$, we obtain, $\boldsymbol{x} \in \operatorname{zer}(\boldsymbol{A}+\boldsymbol{B})$ [2, Proposition 20.33(ii)]. Altogether, it follows [10, Lemma 2.3(ii)] and [10, Theorem 3.3] that $\boldsymbol{x}_{n} \rightarrow \overline{\boldsymbol{x}}$ and hence that $\boldsymbol{p}_{n} \rightarrow \overline{\boldsymbol{x}}$ by (i).

(iii)(a) Since $\boldsymbol{A}$ and $\boldsymbol{B}$ are maximally monotone and $\operatorname{dom} \boldsymbol{B}=\mathcal{K}, \boldsymbol{A}+\boldsymbol{B}$ is maximally monotone [2, Corollary 24.4(i)], $\operatorname{zer}(\boldsymbol{A}+\boldsymbol{B})$ is therefore closed [2, Proposition 23.39]. Hence, the claims follow from (i), (3.16), and [10, Proposition 3.4]. 
(iii)(b): By (i), $\boldsymbol{x}_{n} \rightarrow \overline{\boldsymbol{x}}$, and hence (3.20) implies that $\widetilde{\boldsymbol{p}}_{n} \rightarrow \overline{\boldsymbol{x}}$. Furthermore, it follows from (3.5) that $\boldsymbol{u}_{n} \rightarrow 0$. Hence, since $(\forall n \in \mathbb{N})\left(\widetilde{\boldsymbol{p}}_{n}, \boldsymbol{u}_{n}\right) \in \operatorname{gra}(\boldsymbol{A}+\boldsymbol{B})$ and since $\boldsymbol{A}+\boldsymbol{B}$ is demiregular at $\overline{\boldsymbol{x}}$, by [1, Definition 2.3], $\widetilde{\boldsymbol{p}}_{n} \rightarrow \overline{\boldsymbol{x}}$, and therefore (3.20) implies that $\boldsymbol{x}_{n} \rightarrow \overline{\boldsymbol{x}}$.

(iii)(c) If $\boldsymbol{A}$ or $\boldsymbol{B}$ is uniformly monotone at $\overline{\boldsymbol{x}}$, then $\boldsymbol{A}+\boldsymbol{B}$ is uniformly monotone at $\bar{x}$. Therefore, the result follows from [1, Proposition 2.4(i)].

(iii)(d) Suppose that $\boldsymbol{z} \in \operatorname{int} \operatorname{zer}(\boldsymbol{A}+\boldsymbol{B})$ and fix $\rho \in] 0,+\infty[$ such that $B(\boldsymbol{z} ; \rho) \subset \operatorname{zer}(\boldsymbol{A}+\boldsymbol{B})$. It follows from (3.16) and [10, Proposition 3.2] that

$$
\varepsilon=\sup _{\boldsymbol{x} \in B(\boldsymbol{z} ; \rho)} \sup _{n \in \mathbb{N}}\|\| \boldsymbol{x}_{n}-\boldsymbol{x}\|\|_{\boldsymbol{U}_{n}^{-1}} \leq(1 / \sqrt{\alpha})\left(\sup _{n \in \mathbb{N}}\|\| \boldsymbol{x}_{n}-\boldsymbol{z}\|\| \mid+\sup _{\boldsymbol{x} \in B(\boldsymbol{z} ; \rho)}\|\| \boldsymbol{x}-\boldsymbol{z}\|\|\right)<+\infty
$$

and from (3.16) that

$$
(\forall n \in \mathbb{N})(\forall \boldsymbol{x} \in B(\boldsymbol{z} ; \rho))\|\| \boldsymbol{x}_{n+1}-\boldsymbol{x}\|\|_{\boldsymbol{U}_{n+1}^{-1}}^{2} \leq\|\| \boldsymbol{x}_{n}-\boldsymbol{x}\|\|_{\boldsymbol{U}_{n}^{-1}}^{2}+2 \varepsilon\left(\varepsilon \eta_{n}+\varepsilon_{n}\right)+\left(\varepsilon \eta_{n}+\varepsilon_{n}\right)^{2} .
$$

Hence, the claim follows from (i)], [10, Lemma 2.1], and [10, Proposition 4.3].

Remark 3.2 Here are some remarks.

(i) In the case when $(\forall n \in \mathbb{N}) \boldsymbol{U}_{n}=\mathbf{I d}$, the standard forward-backward-forward splitting algorithm (3.2) reduces to algorithm proposed in [4, Eq. (2.3)], which was proposed initially in the error-free setting in [19].

(ii) An alternative variable metric splitting algorithm proposed in [14] can be used to find a zero of the sum of a maximally monotone operator $\boldsymbol{A}$ and a Lipschitzian monotone operator $\boldsymbol{B}$ in instance when $\mathcal{K}$ is finite-dimensional. This algorithm uses a different error model and involves more iteration-dependent variables than (3.2).

Example 3.3 Let $\boldsymbol{f}: \mathcal{K} \rightarrow[-\infty,+\infty]$ be a proper lower semicontinuous convex function, let $\alpha \in] 0,+\infty[$, let $\beta \in] 0,+\infty[$, let $\boldsymbol{B}: \mathcal{K} \rightarrow \mathcal{K}$ be a monotone and $\beta$-Lipschitzian operator, let $\left(\eta_{n}\right)_{n \in \mathbb{N}} \in \ell_{+}^{1}(\mathbb{N})$, and let $\left(\boldsymbol{U}_{n}\right)_{n \in \mathbb{N}}$ be a sequence in $\mathcal{P}_{\alpha}(\mathcal{K})$ that satisfies (3.1). Furthermore, let $\boldsymbol{x}_{0} \in \mathcal{K}$, let $\left.\varepsilon \in\right] 0, \min \{1,1 /(\mu \beta+1)\}$ [, where $\mu$ is defined as in (3.1), let $\left(\gamma_{n}\right)_{n \in \mathbb{N}}$ be a sequence in $[\varepsilon,(1-\varepsilon) /(\beta \mu)]$. Suppose that the variational inequality

$$
\text { find } \quad \overline{\boldsymbol{x}} \in \mathcal{K} \quad \text { such that } \quad(\forall \boldsymbol{y} \in \mathcal{K}) \quad\langle\overline{\boldsymbol{x}}-\boldsymbol{y} \mid \boldsymbol{B} \overline{\boldsymbol{x}}\rangle+\boldsymbol{f}(\overline{\boldsymbol{x}}) \leq \boldsymbol{f}(\boldsymbol{y})
$$

admits at least one solution and set

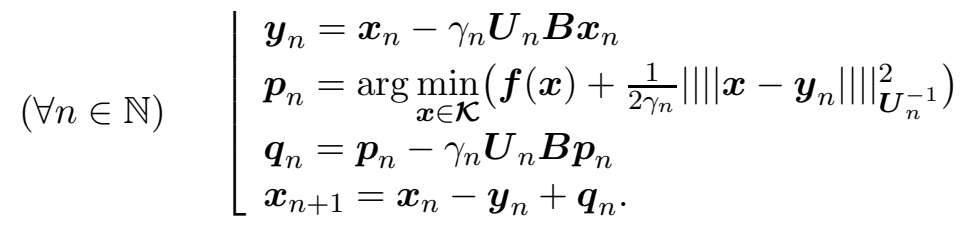

Then $\left(\boldsymbol{x}_{n}\right)_{n \in \mathbb{N}}$ converges weakly to a solution $\overline{\boldsymbol{x}}$ to (3.25).

Proof. Set $\boldsymbol{A}=\partial \boldsymbol{f}$ and $(\forall n \in \mathbb{N}) \boldsymbol{a}_{n}=0, \boldsymbol{b}_{n}=0, \boldsymbol{c}_{n}=0$ in Theorem 3.1](ii), 


\section{Monotone inclusions involving Lipschitzian operators}

The applications of the forward-backward-forward splitting algorithm considered in [4, 9, 19] can be extended to a variable metric setting using Theorem 3.1. As an illustration, we present a variable metric version of the algorithm proposed in [9, Eq. (3.1)]. Recall that the parallel sum of $A: \mathcal{H} \rightarrow 2^{\mathcal{H}}$ and $B: \mathcal{H} \rightarrow 2^{\mathcal{H}}$ is [2]

$$
A \square B=\left(A^{-1}+B^{-1}\right)^{-1} .
$$

Problem 4.1 Let $\mathcal{H}$ be a real Hilbert space, let $m$ be a strictly positive integer, let $z \in \mathcal{H}$, let $A: \mathcal{H} \rightarrow 2^{\mathcal{H}}$ be maximally monotone operator, let $C: \mathcal{H} \rightarrow \mathcal{H}$ be monotone and $\nu_{0}$-Lipschitzian for some $\left.\nu_{0} \in\right] 0,+\infty\left[\right.$. For every $i \in\{1, \ldots, m\}$, let $\mathcal{G}_{i}$ be a real Hilbert space, let $r_{i} \in \mathcal{G}_{i}$, let $B_{i}: \mathcal{G}_{i} \rightarrow 2^{\mathcal{G}_{i}}$ be maximally monotone operator, let $D_{i}: \mathcal{G}_{i} \rightarrow 2^{\mathcal{G}_{i}}$ be monotone and such that $D_{i}^{-1}$ is $\nu_{i}$-Lipschitzian for some $\left.\nu_{i} \in\right] 0,+\infty\left[\right.$, and let $L_{i}: \mathcal{H} \rightarrow \mathcal{G}_{i}$ is a nonzero bounded linear operator. Suppose that

$$
z \in \operatorname{ran}\left(A+\sum_{i=1}^{m} L_{i}^{*}\left(\left(B_{i} \square D_{i}\right)\left(L_{i} \cdot-r_{i}\right)\right)+C\right) .
$$

The problem is to solve the primal inclusion

$$
\text { find } \bar{x} \in \mathcal{H} \text { such that } z \in A \bar{x}+\sum_{i=1}^{m} L_{i}^{*}\left(\left(B_{i} \square D_{i}\right)\left(L_{i} \bar{x}-r_{i}\right)\right)+C \bar{x},
$$

and the dual inclusion

$$
\text { find } \bar{v}_{1} \in \mathcal{G}_{1}, \ldots, \bar{v}_{m} \in \mathcal{G}_{m} \text { such that }(\exists x \in \mathcal{H})\left\{\begin{array}{l}
z-\sum_{i=1}^{m} L_{i}^{*} \bar{v}_{i} \in A x+C x, \\
(\forall i \in\{1, \ldots, m\}) \bar{v}_{i} \in\left(B_{i} \square D_{i}\right)\left(L_{i} x-r_{i}\right) .
\end{array}\right.
$$

As shown in [9], Problem 4.1 covers a wide class of problems in nonlinear analysis and convex optimization problems. However, the algorithm in [9, Theorem 3.1] is studied in the context of a fixed metric. The following result extends this result to a variable metric setting.

Corollary 4.2 Let $\alpha$ be in $] 0,+\infty\left[\right.$, let $\left(\eta_{0, n}\right)_{n \in \mathbb{N}}$ be a sequence in $\ell_{+}^{1}(\mathbb{N})$, let $\left(U_{n}\right)_{n \in \mathbb{N}}$ be a sequence in $\mathcal{P}_{\alpha}(\mathcal{H})$, and for every $i \in\{1, \ldots, m\}$, let $\left(\eta_{i, n}\right)_{n \in \mathbb{N}}$ be a sequence in $\ell_{+}^{1}(\mathbb{N})$, let $\left(U_{i, n}\right)_{n \in \mathbb{N}}$ be a sequence in $\mathcal{P}_{\alpha}\left(\mathcal{G}_{i}\right)$ such that $\mu=\sup _{n \in \mathbb{N}}\left\{\left\|U_{n}\right\|,\left\|U_{1, n}\right\|, \ldots,\left\|U_{m, n}\right\|\right\}<+\infty$ and

$$
(\forall n \in \mathbb{N}) \quad\left(1+\eta_{0, n}\right) U_{n+1} \succcurlyeq U_{n}, \quad \text { and } \quad(\forall i \in\{1, \ldots, m\}) \quad\left(1+\eta_{i, n}\right) U_{i, n+1} \succcurlyeq U_{i, n} .
$$

Let $\left(a_{1, n}\right)_{n \in \mathbb{N}},\left(b_{1, n}\right)_{n \in \mathbb{N}}$, and $\left(c_{1, n}\right)_{n \in \mathbb{N}}$ be absolutely summable sequences in $\mathcal{H}$, and for every $i \in\{1, \ldots, m\}$, let $\left(a_{2, i, n}\right)_{n \in \mathbb{N}},\left(b_{2, i, n}\right)_{n \in \mathbb{N}}$, and $\left(c_{2, i, n}\right)_{n \in \mathbb{N}}$ be absolutely summable sequences in $\mathcal{G}_{i}$. Furthermore, set

$$
\beta=\max \left\{\nu_{0}, \nu_{1}, \ldots, \nu_{m}\right\}+\sqrt{\sum_{i=1}^{m}\left\|L_{i}\right\|^{2}}
$$


let $x_{0} \in \mathcal{H}$, let $\left(v_{1,0}, \ldots, v_{m, 0}\right) \in \mathcal{G}_{1} \oplus \ldots \oplus \mathcal{G}_{m}$, let $\left.\varepsilon \in\right] 0,1 /(1+\beta \mu)\left[\right.$, let $\left(\gamma_{n}\right)_{n \in \mathbb{N}}$ be a sequence in $[\varepsilon,(1-\varepsilon) /(\beta \mu)]$. Set

$$
\left|\begin{array}{l}
y_{1, n}=x_{n}-\gamma_{n} U_{n}\left(C x_{n}+\sum_{i=1}^{m} L_{i}^{*} v_{i, n}+a_{1, n}\right) \\
p_{1, n}=J_{\gamma_{n} U_{n} A}\left(y_{1, n}+\gamma_{n} U_{n} z\right)+b_{1, n} \\
\text { for } i=1, \ldots, m
\end{array}\right| \begin{aligned}
& y_{2, i, n}=v_{i, n}+\gamma_{n} U_{i, n}\left(L_{i} x_{n}-D_{i}^{-1} v_{i, n}+a_{2, i, n}\right) \\
& p_{2, i, n}=J_{\gamma_{n} U_{i, n} B_{i}^{-1}\left(y_{2, i, n}-\gamma_{n} U_{i, n} r_{i}\right)+b_{2, i, n}} \\
& q_{2, i, n}=p_{2, i, n}+\gamma_{n} U_{i, n}\left(L_{i} p_{1, n}-D_{i}^{-1} p_{2, i, n}+c_{2, i, n}\right) \\
& v_{i, n+1}=v_{i, n}-y_{2, i, n}+q_{2, i, n} \\
& q_{1, n}=p_{1, n}-\gamma_{n} U_{n}\left(C p_{1, n}+\sum_{i=1}^{m} L_{i}^{*} p_{2, i, n}+c_{1, n}\right) \\
& x_{n+1}=x_{n}-y_{1, n}+q_{1, n} .
\end{aligned}
$$

Then the following hold.

(i) $\sum_{n \in \mathbb{N}}\left\|x_{n}-p_{1, n}\right\|^{2}<+\infty$ and $(\forall i \in\{1, \ldots, m\}) \sum_{n \in \mathbb{N}}\left\|v_{i, n}-p_{2, i, n}\right\|^{2}<+\infty$.

(ii) There exist a solution $\bar{x}$ to (4.3) and a solution $\left(\bar{v}_{1}, \ldots, \bar{v}_{m}\right)$ to (4.4) such that the following hold.

(a) $x_{n} \rightarrow \bar{x}$ and $p_{1, n} \rightarrow \bar{x}$.

(b) $(\forall i \in\{1, \ldots, m\}) v_{i, n} \rightarrow \bar{v}_{i}$ and $p_{2, i, n} \rightarrow \bar{v}_{i}$.

(c) Suppose that $A$ or $C$ is uniformly monotone at $\bar{x}$, then $x_{n} \rightarrow \bar{x}$ and $p_{1, n} \rightarrow \bar{x}$.

(d) Suppose that $B_{j}^{-1}$ or $D_{j}^{-1}$ is uniformly monotone at $\bar{v}_{j}$, for some $j \in\{1, \ldots, m\}$, then $v_{j, n} \rightarrow \bar{v}_{j}$ and $p_{2, j, n} \rightarrow \bar{v}_{j}$.

Proof. All sequences generated by algorithm (4.7) are well defined by [11, Lemma 3.7]. We define $\mathcal{K}=\mathcal{H} \oplus \mathcal{G}_{1} \oplus \cdots \oplus \mathcal{G}_{m}$ the Hilbert direct sum of the Hilbert spaces $\mathcal{H}$ and $\left(\mathcal{G}_{i}\right)_{1 \leq i \leq m}$, the scalar product and the associated norm of $\mathcal{K}$ respectively defined by

$$
\langle\langle\langle\cdot \mid \cdot\rangle\rangle\rangle:((x, \boldsymbol{v}),(y, \boldsymbol{w})) \mapsto\langle x \mid y\rangle+\sum_{i=1}^{m}\left\langle v_{i} \mid w_{i}\right\rangle \quad \text { and } \quad\|\| \cdot\|\| \|:(x, \boldsymbol{v}) \mapsto \sqrt{\|x\|^{2}+\sum_{i=1}^{m}\left\|v_{i}\right\|^{2}}
$$

where $\boldsymbol{v}=\left(v_{1}, \ldots, v_{m}\right)$ and $\boldsymbol{w}=\left(w_{1}, \ldots, w_{m}\right)$ are generic elements in $\mathcal{G}_{1} \oplus \cdots \oplus \mathcal{G}_{m}$. Set

$$
\left\{\begin{array}{l}
\boldsymbol{A}: \mathcal{K} \rightarrow 2^{\mathcal{K}}:\left(x, v_{1}, \ldots, v_{m}\right) \mapsto(-z+A x) \times\left(r_{1}+B_{1}^{-1} v_{1}\right) \times \ldots \times\left(r_{m}+B_{m}^{-1} v_{m}\right) \\
\boldsymbol{B}: \mathcal{K} \rightarrow \mathcal{K}:\left(x, v_{1}, \ldots, v_{m}\right) \mapsto\left(C x+\sum_{i=1}^{m} L_{i}^{*} v_{i}, D_{1}^{-1} v_{1}-L_{1} x, \ldots, D_{m}^{-1} v_{m}-L_{m} x\right) \\
(\forall n \in \mathbb{N}) \quad \boldsymbol{U}_{n}: \mathcal{K} \rightarrow \mathcal{K}:\left(x, v_{1}, \ldots, v_{m}\right) \mapsto\left(U_{n} x, U_{1, n} v_{1}, \ldots U_{m, n} v_{m}\right) .
\end{array}\right.
$$

Since $\boldsymbol{A}$ is maximally monotone [2, Propositions 20.22 and 20.23], $\boldsymbol{B}$ is monotone and $\beta$ Lipschitzian [9, Eq. (3.10)] with $\operatorname{dom} \boldsymbol{B}=\mathcal{K}, \boldsymbol{A}+\boldsymbol{B}$ is maximally monotone [2, Corollary 24.24(i)]. Now set $(\forall n \in \mathbb{N}) \eta_{n}=\max \left\{\eta_{0, n}, \eta_{1, n}, \ldots, \eta_{m, n}\right\}$. Then $\left(\eta_{n}\right)_{n \in \mathbb{N}} \in \ell_{+}^{1}(\mathbb{N})$. Moreover, we derive from our assumptions on the sequences $\left(U_{n}\right)_{n \in \mathbb{N}}$ and $\left(U_{1, n}\right)_{n \in \mathbb{N}}, \ldots,\left(U_{m, n}\right)_{n \in \mathbb{N}}$ that

$$
\mu=\sup _{n \in \mathbb{N}}\left\|\boldsymbol{U}_{n}\right\|<+\infty \quad \text { and } \quad\left(1+\eta_{n}\right) \boldsymbol{U}_{n+1} \succcurlyeq \boldsymbol{U}_{n} \in \mathcal{P}_{\alpha}(\mathcal{K})
$$


In addition, 22, Propositions 23.15(ii) and 23.16] yield $(\forall \gamma \in] 0,+\infty[)(\forall n \in \mathbb{N})\left(\forall\left(x, v_{1}, \ldots, v_{m}\right) \in\right.$ $\mathcal{K})$

$$
J_{\gamma \boldsymbol{U}_{n} \boldsymbol{A}}\left(x, v_{1}, \ldots, v_{m}\right)=\left(J_{\gamma U_{n} A}\left(x+\gamma U_{n} z\right),\left(J_{\gamma U_{i, n} B_{i}^{-1}}\left(v_{i}-\gamma U_{i, n} r_{i}\right)\right)_{1 \leq i \leq m}\right) .
$$

It is shown in [9, Eq. (3.12)] and [9, Eq. (3.13)] that under the condition (4.2), $\operatorname{zer}(\boldsymbol{A}+\boldsymbol{B}) \neq \varnothing$. Moreover, [9, Eq. (3.21)] and [9, Eq. (3.22)] yield

$$
\left(\bar{x}, \bar{v}_{1}, \ldots, \bar{v}_{m}\right) \in \operatorname{zer}(\boldsymbol{A}+\boldsymbol{B}) \Rightarrow \bar{x} \text { solves (4.3) and }\left(\bar{v}_{1}, \ldots, \bar{v}_{m}\right) \text { solves (4.4). }
$$

Let us next set

$$
(\forall n \in \mathbb{N}) \quad\left\{\begin{array} { l } 
{ \boldsymbol { x } _ { n } = ( x _ { n } , v _ { 1 , n } , \ldots , v _ { m , n } ) } \\
{ \boldsymbol { y } _ { n } = ( y _ { 1 , n } , y _ { 2 , 1 , n } , \ldots , y _ { 2 , m , n } ) } \\
{ \boldsymbol { p } _ { n } = ( p _ { 1 , n } , p _ { 2 , 1 , n } , \ldots , p _ { 2 , m , n } ) } \\
{ \boldsymbol { q } _ { n } = ( q _ { 1 , n } , q _ { 2 , 1 , n } , \ldots , q _ { 2 , m , n } ) }
\end{array} \quad \text { and } \quad \left\{\begin{array}{l}
\boldsymbol{a}_{n}=\left(a_{1, n}, a_{2,1, n}, \ldots, a_{2, m, n}\right) \\
\boldsymbol{b}_{n}=\left(b_{1, n}, b_{2,1, n}, \ldots, b_{2, m, n}\right) \\
\boldsymbol{c}_{n}=\left(c_{1, n}, c_{2,1, n}, \ldots, c_{2, m, n}\right) .
\end{array}\right.\right.
$$

Then our assumptions imply that

$$
\sum_{n \in \mathbb{N}}|||| \boldsymbol{a}_{n}||||<\infty, \quad \sum_{n \in \mathbb{N}}|||| \boldsymbol{b}_{n}|| \| \mid<\infty, \quad \text { and } \quad \sum_{n \in \mathbb{N}}\left|\left\||| \boldsymbol{c}_{n}|\||<\infty .\right.\right.
$$

Furthermore, it follows from the definition of $\boldsymbol{B}$, (4.11), and (4.13) that (4.7) can be rewritten in $\mathcal{K}$ as

$$
(\forall n \in \mathbb{N}) \quad \mid \begin{aligned}
& \boldsymbol{y}_{n}=\boldsymbol{x}_{n}-\gamma_{n} \boldsymbol{U}_{n}\left(\boldsymbol{B} \boldsymbol{x}_{n}+\boldsymbol{a}_{n}\right) \\
& \boldsymbol{p}_{n}=J_{\gamma_{n}} \boldsymbol{U}_{n} \boldsymbol{A} \boldsymbol{y}_{n}+\boldsymbol{b}_{n} \\
& \boldsymbol{q}_{n}=\boldsymbol{p}_{n}-\gamma_{n} \boldsymbol{U}_{n}\left(\boldsymbol{B} \boldsymbol{p}_{n}+\boldsymbol{c}_{n}\right) \\
& \boldsymbol{x}_{n+1}=\boldsymbol{x}_{n}-\boldsymbol{y}_{n}+\boldsymbol{q}_{n}
\end{aligned}
$$

which is (3.2). Moreover, every specific conditions in Theorem 3.1 are satisfied.

(i): By Theorem 3.1][i), $\sum_{n \in \mathbb{N}}\left|\left\|\mid \boldsymbol{x}_{n}-\boldsymbol{p}_{n}\right\| \|^{2}<\infty\right.$.

(ii)(a) \&(ii)(b), These assertions follow from Theorem 3.1](ii).

(ii)(c): Theorem 3.1](ii)] shows that $\left(\bar{x}, \bar{v}_{1}, \ldots, \bar{v}_{m}\right) \in \operatorname{zer}(\boldsymbol{A}+\boldsymbol{B})$. Hence, it follows from [9, $\mathrm{Eq}(3.19)]$ that $\left(\bar{x}, \bar{v}_{1}, \ldots, \bar{v}_{m}\right)$ satisfies the inclusions

$$
\left\{\begin{array}{l}
-\sum_{i=1}^{m} L_{i}^{*} \bar{v}_{i}-C \bar{x} \in-z+A \bar{x} \\
(\forall i \in\{1, \ldots, m\}) L_{i} \bar{x}-D_{i}^{-1} \bar{v}_{i} \in r_{i}+B_{i}^{-1} \bar{v}_{i} .
\end{array}\right.
$$

For every $n \in \mathbb{N}$ and every $i \in\{1, \ldots, m\}$, set

$$
\left\{\begin{array} { l } 
{ \widetilde { y } _ { 1 , n } = x _ { n } - \gamma _ { n } U _ { n } ( C x _ { n } + \sum _ { i = 1 } ^ { m } L _ { i } ^ { * } v _ { i , n } ) } \\
{ \widetilde { p } _ { 1 , n } = J _ { \gamma _ { n } U _ { n } A } ( \widetilde { y } _ { 1 , n } + \gamma _ { n } U _ { n } z ) }
\end{array} \quad \text { and } \quad \left\{\begin{array}{l}
\widetilde{y}_{2, i, n}=v_{i, n}+\gamma_{n} U_{i, n}\left(L_{i} x_{n}-D_{i}^{-1} v_{i, n}\right) \\
\widetilde{p}_{2, i, n}=J_{\gamma_{n} U_{i, n} B_{i}^{-1}}\left(\widetilde{y}_{2, i, n}-\gamma_{n} U_{i, n} r_{i}\right) .
\end{array}\right.\right.
$$


Then, using [11, Lemma 3.7], we get

$$
\widetilde{p}_{1, n}-p_{1, n} \rightarrow 0 \quad \text { and } \quad(\forall i \in\{1, \ldots, m\}) \quad \widetilde{p}_{2, i, n}-p_{2, i, n} \rightarrow 0,
$$

in turn, by (i)[(ii)(a), and (ii)(b), we obtain

$$
\widetilde{p}_{1, n}-x_{n} \rightarrow 0, \quad \widetilde{p}_{1, n} \rightarrow \bar{x}, \quad \text { and } \quad(\forall i \in\{1, \ldots, m\}) \quad \tilde{p}_{2, i, n}-v_{i, n} \rightarrow 0, \quad \widetilde{p}_{2, i, n} \rightarrow \bar{v}_{i} .
$$

Furthermore, we derive from (4.17) that

$$
(\forall n \in \mathbb{N})\left\{\begin{array}{l}
\gamma_{n}^{-1} U_{n}^{-1}\left(x_{n}-\widetilde{p}_{1, n}\right)-\sum_{i=1}^{m} L_{i}^{*} v_{i, n}-C x_{n} \in-z+A \widetilde{p}_{1, n} \\
(\forall i \in\{1, \ldots, m\}) \gamma_{n}^{-1} U_{i, n}^{-1}\left(v_{i, n}-\widetilde{p}_{2, i, n}\right)+L_{i} x_{n}-D_{i}^{-1} v_{i, n} \in r_{i}+B_{i}^{-1} \widetilde{p}_{2, i, n} .
\end{array}\right.
$$

Since $A$ is uniformly monotone at $\bar{x}$, using (4.16) and (4.20), there exists an increasing function $\phi_{A}:[0,+\infty[\rightarrow[0,+\infty]$ vanishing only at 0 such that, for every $n \in \mathbb{N}$,

$$
\begin{aligned}
\phi_{A}\left(\left\|\widetilde{p}_{1, n}-\bar{x}\right\|\right) & \leqslant\left\langle\widetilde{p}_{1, n}-\bar{x} \mid \gamma_{n}^{-1} U_{n}^{-1}\left(x_{n}-\widetilde{p}_{1, n}\right)-\sum_{i=1}^{m}\left(L_{i}^{*} v_{i, n}-L_{i}^{*} \bar{v}_{i}\right)-\left(C x_{n}-C \bar{x}\right)\right\rangle \\
& =\left\langle\widetilde{p}_{1, n}-\bar{x} \mid \gamma_{n}^{-1} U_{n}^{-1}\left(x_{n}-\widetilde{p}_{1, n}\right)\right\rangle-\sum_{i=1}^{m}\left\langle\widetilde{p}_{1, n}-\bar{x} \mid L_{i}^{*} v_{i, n}-L_{i}^{*} \bar{v}_{i}\right\rangle-\chi_{n}
\end{aligned}
$$

where we denote $(\forall n \in \mathbb{N}) \chi_{n}=\left\langle\widetilde{p}_{1, n}-\bar{x} \mid C x_{n}-C \bar{x}\right\rangle$. Since $\left(B_{i}^{-1}\right)_{1 \leq i \leq m}$ are monotone, for every $i \in\{1, \ldots, m\}$, we obtain

$$
\begin{aligned}
(\forall n \in \mathbb{N}) \quad 0 & \leqslant\left\langle\widetilde{p}_{2, i, n}-\bar{v}_{i} \mid L_{i} x_{n}+\gamma_{n}^{-1} U_{i, n}^{-1}\left(v_{i, n}-\widetilde{p}_{2, i, n}\right)-L_{i} \bar{x}-\left(D_{i}^{-1} v_{i, n}-D_{i}^{-1} \bar{v}_{i}\right)\right\rangle \\
& =\left\langle\widetilde{p}_{2, i, n}-\bar{v}_{i} \mid L_{i}\left(x_{n}-\bar{x}\right)+\gamma_{n}^{-1} U_{i, n}^{-1}\left(v_{i, n}-\widetilde{p}_{2, i, n}\right)\right\rangle-\beta_{i, n}
\end{aligned}
$$

where $(\forall n \in \mathbb{N}) \beta_{i, n}=\left\langle\widetilde{p}_{2, i, n}-\bar{v}_{i} \mid D_{i}^{-1} v_{i, n}-D_{i}^{-1} \bar{v}_{i}\right\rangle$. Now, adding (4.22) from $i=1$ to $i=m$ and (4.21), we obtain, for every $n \in \mathbb{N}$,

$$
\begin{aligned}
\phi_{A}\left(\left\|\widetilde{p}_{1, n}-\bar{x}\right\|\right) \leq & \left\langle\widetilde{p}_{1, n}-\bar{x} \mid \gamma_{n}^{-1} U_{n}^{-1}\left(x_{n}-\widetilde{p}_{1, n}\right)\right\rangle+\left\langle\widetilde{p}_{1, n}-\bar{x} \mid \sum_{i=1}^{m} L_{i}^{*}\left(\widetilde{p}_{2, i, n}-v_{i, n}\right)\right\rangle \\
& +\sum_{i=1}^{m}\left\langle\widetilde{p}_{2, i, n}-\bar{v}_{i} \mid L_{i}\left(x_{n}-\widetilde{p}_{1, n}\right)+\gamma_{n}^{-1} U_{i, n}^{-1}\left(v_{i, n}-\widetilde{p}_{2, i, n}\right)\right\rangle-\chi_{n}-\sum_{i=1}^{m} \beta_{i, n} .
\end{aligned}
$$

For every $n \in \mathbb{N}$ and every $i \in\{1, \ldots, m\}$, we expand $\chi_{n}$ and $\beta_{i, n}$ as

$$
\left\{\begin{array}{l}
\chi_{n}=\left\langle x_{n}-\bar{x} \mid C x_{n}-C \bar{x}\right\rangle+\left\langle\widetilde{p}_{1, n}-x_{n} \mid C x_{n}-C \bar{x}\right\rangle \\
\beta_{i, n}=\left\langle v_{i, n}-\bar{v}_{i} \mid D_{i}^{-1} v_{i, n}-D_{i}^{-1} \bar{v}_{i}\right\rangle+\left\langle\widetilde{p}_{2, i, n}-v_{i, n} \mid D_{i}^{-1} v_{i, n}-D_{i}^{-1} \bar{v}_{i}\right\rangle .
\end{array}\right.
$$

By monotonicity of $C$ and $\left(D_{i}^{-1}\right)_{1 \leq i \leq m}$,

$$
(\forall n \in \mathbb{N}) \quad\left\{\begin{array}{l}
\left\langle x_{n}-\bar{x} \mid C x_{n}-C \bar{x}\right\rangle \geq 0 \\
(\forall i \in\{1, \ldots, m\})\left\langle v_{i, n}-\bar{v}_{i} \mid D_{i}^{-1} v_{i, n}-D_{i}^{-1} \bar{v}_{i}\right\rangle \geq 0 .
\end{array}\right.
$$


Therefore, for every $n \in \mathbb{N}$, we derive from (4.24) and (4.23) that

$$
\begin{aligned}
\phi_{A}\left(\left\|\widetilde{p}_{1, n}-\bar{x}\right\|\right) \leq & \phi_{A}\left(\left\|\widetilde{p}_{1, n}-\bar{x}\right\|\right)+\left\langle x_{n}-\bar{x} \mid C x_{n}-C \bar{x}\right\rangle+\sum_{i=1}^{m}\left\langle v_{i, n}-\bar{v}_{i} \mid D_{i}^{-1} v_{i, n}-D_{i}^{-1} \bar{v}_{i}\right\rangle \\
\leq & \left\langle\widetilde{p}_{1, n}-\bar{x} \mid \gamma_{n}^{-1} U_{n}^{-1}\left(x_{n}-\widetilde{p}_{1, n}\right)\right\rangle+\left\langle\widetilde{p}_{1, n}-\bar{x} \mid \sum_{i=1}^{m} L_{i}^{*}\left(\widetilde{p}_{2, i, n}-v_{i, n}\right)\right\rangle \\
& +\sum_{i=1}^{m}\left\langle\widetilde{p}_{2, i, n}-\bar{v}_{i} \mid L_{i}\left(x_{n}-\widetilde{p}_{1, n}\right)+\gamma_{n}^{-1} U_{i, n}^{-1}\left(v_{i, n}-\widetilde{p}_{2, i, n}\right)\right\rangle \\
& -\left\langle\widetilde{p}_{1, n}-x_{n} \mid C x_{n}-C \bar{x}\right\rangle-\sum_{i=1}^{m}\left\langle\widetilde{p}_{2, i, n}-v_{i, n} \mid D_{i}^{-1} v_{i, n}-D_{i}^{-1} \bar{v}_{i}\right\rangle
\end{aligned}
$$

We set

$$
\zeta=\max _{1 \leq i \leq m} \sup _{n \in \mathbb{N}}\left\{\left\|x_{n}-\bar{x}\right\|,\left\|\widetilde{p}_{1, n}-\bar{x}\right\|,\left\|v_{i, n}-\bar{v}_{i}\right\|,\left\|\widetilde{p}_{2, i, n}-\bar{v}_{i}\right\|\right\} .
$$

Then it follows from (ii)(a), (ii)(b), and (4.19) that $\zeta<\infty$, and from [10, Lemma 2.1(ii)] that $(\forall n \in \mathbb{N})\left\|\gamma_{n}^{-1} U_{n}^{-1}\right\| \leq(\varepsilon \alpha)^{-1}$ and $(\forall i \in\{1, \ldots, m\})\left\|\gamma_{n}^{-1} U_{i, n}^{-1}\right\| \leq(\varepsilon \alpha)^{-1}$. Therefore, using the Cauchy-Schwarz inequality, and the Lipschitzianity of $C$ and $\left(D_{i}^{-1}\right)_{1 \leq i \leq m}$, we derive from (4.26) that

$$
\begin{aligned}
\phi_{A}\left(\left\|\widetilde{p}_{1, n}-\bar{x}\right\|\right) \leq & (\varepsilon \alpha)^{-1} \zeta\left\|x_{n}-\widetilde{p}_{1, n}\right\|+\zeta \sum_{i=1}^{m}\left(\left\|L_{i}\right\|\left\|x_{n}-\widetilde{p}_{1, n}\right\|+(\varepsilon \alpha)^{-1}\left\|v_{i, n}-\widetilde{p}_{2, i, n}\right\|\right) \\
& +\zeta\left(\sum_{i=1}^{m}\left\|L_{i}^{*}\right\|\left\|\widetilde{p}_{2, i, n}-v_{i, n}\right\|+\nu_{0}\left\|\widetilde{p}_{1, n}-x_{n}\right\|+\sum_{i=1}^{m} \nu_{i}\left\|\widetilde{p}_{2, i, n}-v_{i, n}\right\|\right) \\
\rightarrow & 0 .
\end{aligned}
$$

We deduce from (4.28) and (4.19) that $\phi_{A}\left(\left\|\widetilde{p}_{1, n}-\bar{x}\right\|\right) \rightarrow 0$, which implies that $\widetilde{p}_{1, n} \rightarrow \bar{x}$. In turn, $x_{n} \rightarrow \bar{x}$ and $p_{n} \rightarrow \bar{x}$. Likewise, if $C$ is uniformly monotone at $\bar{x}$, there exists an increasing function $\phi_{C}:[0,+\infty[\rightarrow[0,+\infty]$ that vanishes only at 0 such that

$$
\begin{aligned}
\phi_{C}\left(\left\|x_{n}-\bar{x}\right\|\right) \leq & (\varepsilon \alpha)^{-1} \zeta\left\|x_{n}-\widetilde{p}_{1, n}\right\|+\zeta \sum_{i=1}^{m}\left(\left\|L_{i}\right\|\left\|x_{n}-\widetilde{p}_{1, n}\right\|+(\varepsilon \alpha)^{-1}\left\|v_{i, n}-\widetilde{p}_{2, i, n}\right\|\right) \\
& +\zeta\left(\sum_{i=1}^{m}\left\|L_{i}^{*}\right\|\left\|\widetilde{p}_{2, i, n}-v_{i, n}\right\|+\nu_{0}\left\|\widetilde{p}_{1, n}-x_{n}\right\|+\sum_{i=1}^{m} \nu_{i}\left\|\widetilde{p}_{2, i, n}-v_{i, n}\right\|\right) \\
\rightarrow & 0,
\end{aligned}
$$

in turn, $x_{n} \rightarrow \bar{x}$ and $p_{n} \rightarrow \bar{x}$.

(ii)(d) Proceeding as in the proof of (ii)(c), we obtain the conclusions.

Acknowledgement. I thank Professor Patrick L. Combettes for bringing this problem to my attention and for helpful discussions. 


\section{References}

[1] H. Attouch, L. M. Briceño-Arias, and P. L. Combettes, A parallel splitting method for coupled monotone inclusions, SIAM J. Control Optim., vol. 48, pp. 3246-3270, 2010.

[2] H. H. Bauschke and P. L. Combettes, Convex Analysis and Monotone Operator Theory in Hilbert Spaces. Springer, New York, 2011.

[3] J. F. Bonnans, J. Ch. Gilbert, C. Lemaréchal, and C. A. Sagastizábal, A family of variable metric proximal methods, Math. Programming, vol. 68, pp. 15-47, 1995.

[4] L. M. Briceño-Arias and P. L. Combettes, A monotone+skew splitting model for composite monotone inclusions in duality, SIAM J. Optim., vol. 21, pp. 1230-1250, 2011.

[5] J. V. Burke and M. Qian, A variable metric proximal point algorithm for monotone operators, SIAM J. Control Optim., vol. 37, pp. 353-375, 1999.

[6] J. V. Burke and M. Qian, On the superlinear convergence of the variable metric proximal point algorithm using Broyden and BFGS matrix secant updating, Math. Program., vol. 88, pp. 157-181, 2000.

[7] P. L. Combettes, Quasi-Fejérian analysis of some optimization algorithms, in Inherently Parallel Algorithms in Feasibility and Optimization and Their Applications (D. Butnariu, Y. Censor, and S. Reich, Eds.), pp. 115-152. New York: Elsevier, 2001.

[8] P. L. Combettes, Solving monotone inclusions via compositions of nonexpansive averaged operators, Optimization, vol. 53, pp. 475-504, 2004.

[9] P. L. Combettes and J.-C. Pesquet, Primal-dual splitting algorithm for solving inclusions with mixtures of composite, Lipschitzian, and parallel-sum type monotone operators, Set-Valued Var. Anal., vol. 20, pp. 307-330, 2012.

[10] P. L. Combettes and B. C. Vũ, Variable metric quasi-Fejér monotonicity, Nonlinear Anal., to appear. http://www.sciencedirect.com/science/article/pii/S0362546X12003616

[11] P. L. Combettes and B. C. Vũ, Variable metric forward-backward splitting with applications to monotone inclusions in duality, Optimization, to appear. http://arxiv.org/abs/1206.6791

[12] B. Mercier, Topics in Finite Element Solution of Elliptic Problems (Lectures on Mathematics, no. 63). Tata Institute of Fundamental Research, Bombay, 1979.

[13] C. Lemaréchal and C. Sagastizábal, Variable metric bundle methods: from conceptual to implementable forms, Math. Program., vol. 76, pp. 393-410, 1997.

[14] P. A. Lotito, L. A. Parente, and M. V. Solodov, A class of variable metric decomposition methods for monotone variational inclusions, J. Convex Anal., vol. 16, pp. 857-880, 2009.

[15] L. A. Parente, P. A. Lotito, and M. V. Solodov, A class of inexact variable metric proximal point algorithms, SIAM J. Optim., vol. 19, pp. 240-260, 2008. 
[16] L. Qi and X. Chen, A preconditioning proximal Newton's method for nondifferentiable convex optimization, Math. Program., vol. 76, pp. 411-430, 1995.

[17] R. T. Rockafellar, Monotone operators and the proximal point algorithm, SIAM J. Control Optimization, vol. 14, pp. 877-898, 1976.

[18] P. Tseng, Applications of a splitting algorithm to decomposition in convex programming and variational inequalities, SIAM J. Control Optim., vol. 29, pp. 119-138, 1991.

[19] P. Tseng, A modified forward-backward splitting method for maximal monotone mappings, SIAM J. Control Optim., vol. 38, pp. 431-446, 2000. 